\section{Heredero del \\ el cine de Jim Abrahams y de los hermanos Zucker en las comedias de Ben Stiller:} Heir of ZAZ: Jim Abrahams and : Zucker brothers's cinema : in Ben Stiller's comedies :

\author{
Sebastián Chávez ${ }^{1}$, Sebastián Gómez ${ }^{2}$, Daniel Montoya ${ }^{3}$,
} Ana Paula Sánchez ${ }^{4}$, Claudia Torres ${ }^{5}$.

\section{Resumen}

Dos tipos de comedias nos ofrecen, por un lado, el trío integrado por Jim Abrahams, Jerry Zucker y David Zucker y, por el otro, las películas dirigidas por Ben Stiller. Sus trabajos han visto la luz con 20 años de diferencia y se conciben bajo perspectivas del mundo completamente distintas. En ambos casos se parodian situaciones identificables en la realidad pero cada uno lo hace usando el humor dentro de su propio lenguaje y estilo. Los primeros han establecido un culto gracias a una comedia ligada con lo absurdo, pero Ben Stiller usa la sátira para construir el mundo en el que sus historias cobran vida.

\section{Abstract}

Two types of comedy movies offers, on one hand, Jim Abrahams, Jerry Zucker and David Zucker and, on the other, Ben Stiller's films. His works are 20 years apart and conceived under completely different perspectives of the world. In both cases they parody identifiable situations in reality but each one does so using humor within their own language and style. The former have established a cult because comedy is linked to absurd, but Ben Stiller uses satire to build the world in which his stories come alive.

\section{Palabras clave}

Jim Abrahams; David Zucker; Jerry Zucker; Ben Stiller; influencia cinematográfica; parodia; comedia

\section{Key words}

Jim Abrahams; David Zucker; Jerry Zucker; Ben Stiller; cinematographic influence; parody; comedy

1. Universidad Peruana de Ciencias Aplicadas, Programa Académico de Comunicación Audiovisual y Medios Interactivos, e-mail: seb99chg@gmail.com

2. Universidad Peruana de Ciencias Aplicadas, Programa Académico de Comunicación Audiovisual y Medios Interactivos, e-mail: gomezcamposfamilia@hotmail.com

3. Universidad Peruana de Ciencias Aplicadas, Programa Académico de Comunicación Audiovisual y Medios Interactivos, e-mail: danielmontoyaromero2017@gmail.com

4. Universidad Peruana de Ciencias Aplicadas, Programa Académico de Comunicación y Periodismo,e-mail: anapsnchz@gmail.com

5. Universidad Peruana de Ciencias Aplicadas, Programa Académico de Comunicación y Periodismo, e-mail: clau.m.t.c@gmail.com
SGRUPULOS

Volumen 7

Número 2

Julio a diciembre 2019

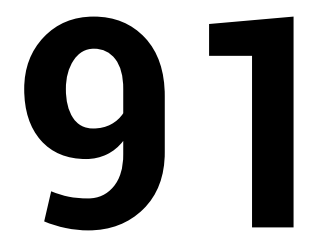

Citar como:

Chávez, S., Gómez, S., Montoya, D., Sánchez,

A., Torres, C. (2019).

Heredero del ZAZ: el cine de Jim Abrahams y de los hermanos Zucker en las comedias de Ben Stiller. CineScrúpulos, 7(2), 91-96.

DOI: https://doi. org/10.19083/

cinescrupulos.v7i2.1264

\section{(c) \\ Recibido: \\ 3 de julio de 2019 \\ Aceptado:}

23 de setiembre de 2019

Publicado:

9 de diciembre de 2019 
scrupulos

Volumen 7

Número 2

Julio a diciembre 2019

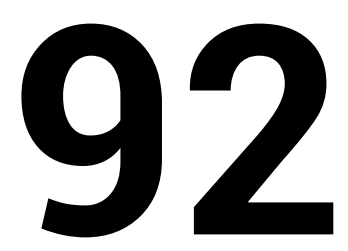

Las historias en

el cine de Jim

Abrahams y de los

hermanos Zucker se

caracterizan por su

tendencia surrealista

y absurda. En el

contexto en el que

se lleva a cabo la

narración no existe

una distinción entre

el hecho cómico y

la normalidad de la

vida. Las situaciones

de torpeza o el

absurdismo se

convierten en

la norma y en lo

cotidiano.
: A través de los años la risa ha sido la mejor medicina para superar

: las adversidades. La Gran Depresión de 1929 dejó un gran vacío en

- la vida de los norteamericanos por la falta de trabajo y los tiempos

: difíciles. Una forma de solución ligera y entretenida se imponía

- y es así como la comedia tuvo relevancia en el mundo de Ho-

: llywood y se convirtió en uno de los géneros cinematográficos por

: excelencia. Antes del sonido, la narrativa en la comedia tuvo como

- base el uso del slapstick y del gag. Con la llegada del cine sonoro,

: los estándares de la comedia americana cambiaron por completo

- al incorporar el diálogo. La sátira, los insultos y las bromas dejaron

: de ser algo que solo podía disfrutarse visualmente. Gracias a esta

: innovación cinematográfica la comedia evolucionó y se consolidaron subgéneros con particularidades distintivas.

$\bullet$

EL ABSURDO FRENTE A LA CRÍTICA

$\bullet$

- Las historias en el cine de Jim Abrahams y de los hermanos Zu-

: cker se caracterizan por su tendencia surrealista y absurda. En el

- contexto en el que se lleva a cabo la narración no existe una dis- tinción entre el hecho cómico y la normalidad de la vida. Las si: tuaciones de torpeza o el absurdismo se convierten en la norma y - en lo cotidiano. El espectador reconoce que lo que pasa no corres- ponde con la realidad sino que es más bien una exageración de la : misma. Esto se observa principalmente en "Airplane!" (Abrahams, Zucker y Zucker, 1980) en la que de forma continua y sin respiro los personajes saltan de situación en situación. En este caso, la : construcción dramática tiene elementos cómicos como el slapstick

: que son llevados al extremo y en el marco de la incongruencia. $\mathrm{Ni}$

: hablar de los tiempos y escenarios que se mezclan entre sí y dotan

- la historia de una mayor carga de absurdo. Por ejemplo, cuando

: el avión está por despegar una joven pareja se despide como si se tratara de un tren que parte.

En "The Naked Gun: From the Files of Police Squad!" (Zucker, - 1988) el detective Drebin es torpe y las situaciones que lo rodean : son poco verosímiles. Se logra identificar una contraparte, un per- sonaje más racional, pero si tomamos en cuenta la ruptura de la - cuarta pared y la película como un todo, permanece en el espec: tador la sensación de acudir a un mundo entorpecido en el que - la razón no prima sobre las acciones de los personajes. Los her- manos Zucker y Jim Abrahams parodian situaciones y personajes, : pero la impresión que dejan sus trabajos en el espectador es la de - una mera burla sin llegar a una construcción crítica.

- La comedia elaborada en las películas de Ben Stiller se percibe más - ligada a la sátira, a diferencia de las parodias anteriormente men: cionadas. La crítica se hace más explícita porque los personajes - están envueltos en situaciones relacionadas con temas políticos y : sociales, realidades que resultan sensibles en el contexto estadou- 
nidense. En "Zoolander" (Stiller, 2001) el conflicto se genera a raíz : de que el primer ministro de Malasia decide hacer una reforma : política en las leyes laborales. Por ello, un prestigioso diseñador : llamado Jacobim Mugatu decide manipular a un modelo cotizado : y usarlo como títere para asesinar al primer ministro. A modo de : sátira se aprecia lo superficial que puede llegar a ser el mundo del modelaje ya que los modelos realizan batallas de catwalk para sa- : ber quién es el mejor y se preocupan únicamente por su apariencia o por mostrar su mejor perfil.

En "Tropic Thunder" (Stiller, 2008) se hace una crítica a la industria cinematográfica y a los estereotipos que se utilizan tanto para los personajes como para las películas de género. Encontramos actores adictos a las drogas, otros que se toman muy en serio sus papeles y : algunos que sienten envidia del éxito ajeno mientras el director, al ser un novato, es incapaz de dirigir adecuadamente a las estrellas y se deja influenciar por cualquiera con una personalidad autoritaria. Respecto a la supuesta película bélica que se filma, no puede faltar una guerra, explosiones por doquier y un sinfín de balas que atra- : viesan el escenario en todo momento.

\section{EN CARNE Y HUESO}

Existe una diferencia abismal en cómo son y cómo tratan a sus personajes cada uno de los directores mencionados. En el cine de los Zucker notamos que todos son tontos porque cada uno de ellos siempre realiza una acción que no tiene sentido dentro de la historia e incluso varios de ellos optan por el slapstick como recurso para hacer reír al espectador. Se nota el uso de una violencia amoral: cuando hacen acciones incorrectas no cargan con un sentido de culpa. Un ejemplo de ello podemos apreciarlo en "Airplane!" (Abrahams, Zucker y Zucker, 1980) cuando todos los pasajeros del avión golpean a una mujer de forma gratuita.

Los personajes que se aprecian en las películas de los hermanos Zucker están envueltos en situaciones disparatadas que no se asemejan con la realidad, por lo que es posible decir que utilizan un humor surrealista. La irracionalidad de los personajes origina gracia en el público y ello genera una homogeneidad ya que todos son tontos, quizás alguno más que otro. Incluso se aprecia un humor organizado de manera rápida y acelerada, como si fueran sketches de una escena humorística. Es lo que sucede cuando Frank Drebin, el personaje principal que es teniente de la policía de Los Ángeles en "The Naked Gun: From the Files of Police Squad!" (Zucker, 1988), es despistado y tonto a lo largo de la trama y en una sucesión de escenas incoherentes.

Este es un factor importante a tener en cuenta porque es bastante diferente a lo que plantea Stiller ya que, en su caso, los persona- :

\section{SGRUPULOS}

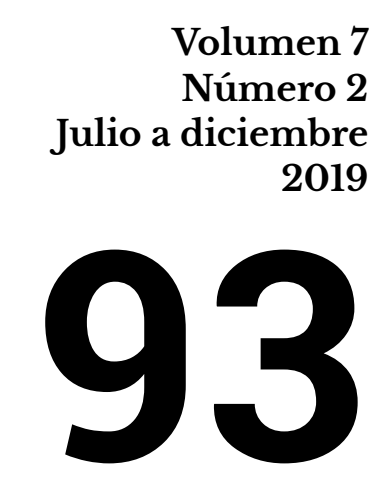

En el cine de los Zucker notamos que todos son tontos porque cada uno de ellos siempre realiza una acción que no tiene sentido dentro de la historia

e incluso varios de ellos optan por el slapstick como recurso para hacer reír al espectador. 
ScRupulos:

Volumen 7

Número 2

Julio a diciembre 2019

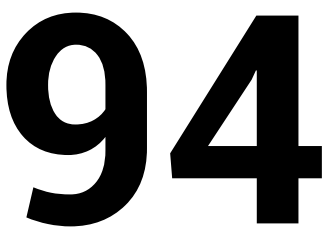

Es cierto que algunos personajes de Stiller son tontos (indiscutiblemente es el caso del propio Derek Zoolander), pero este tipo de arquetipo es típico en las comedias americanas y, como ya se ha mencionado, el enfoque de ambas propuestas es muy diferente como para considerar una influencia.

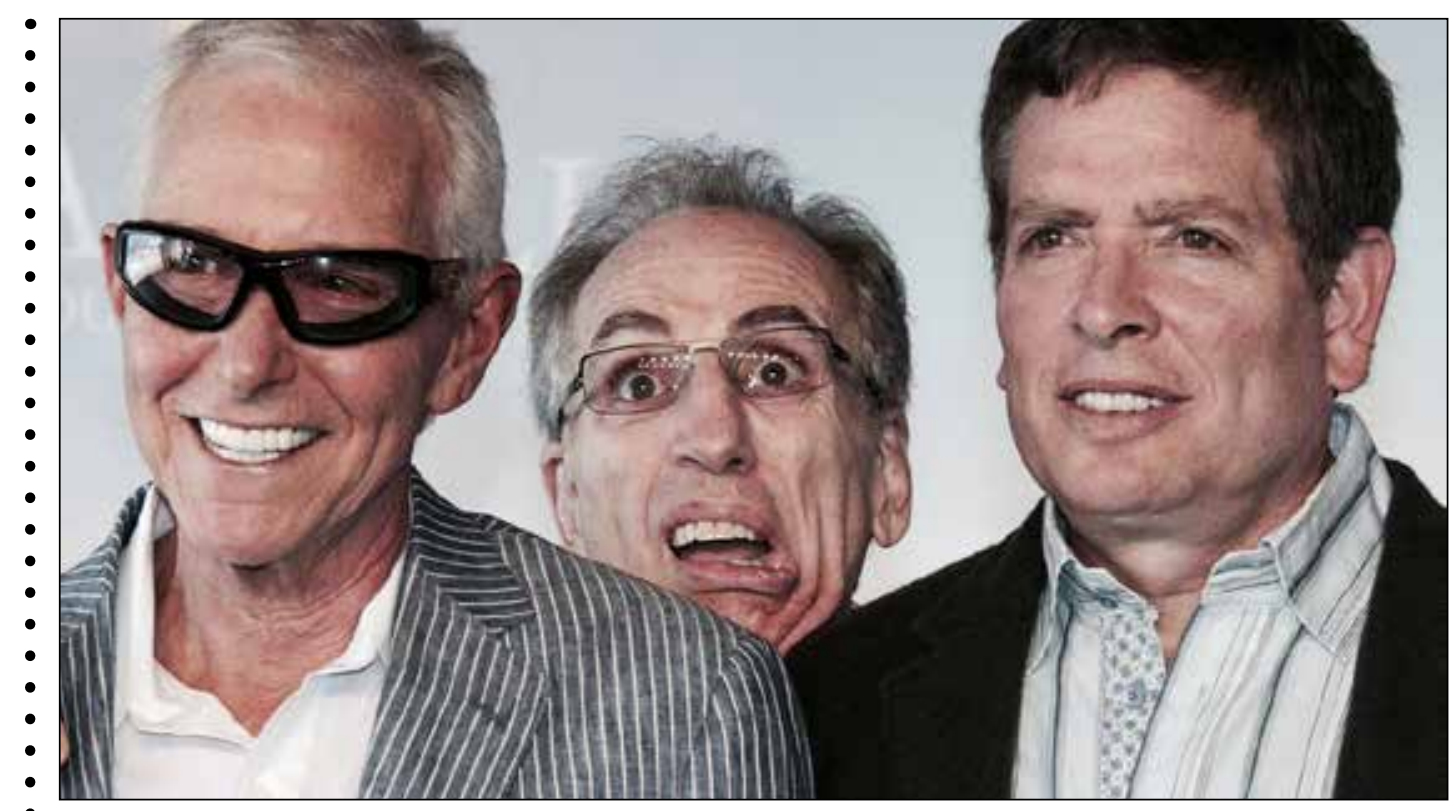

Jim Abrahams, Jerry Zucker y David Zucker.

jes están dotados de personalidades, sentimientos y hasta de un pasado. Stiller los retrata con un trasfondo y esta es la principal - diferencia entre ambos estilos. Las situaciones, en este caso, son - más realistas y construyen un universo con más sentido. Los per: sonajes tienden a ser más individuales o heterogéneos: cada uno - es diferente al momento de interpretar y causar humor en sus - películas. Por eso no está al nivel de los Zucker: porque juegan : en la línea de lo asimilable a la vista del público. Es cierto que - algunos personajes de Stiller son tontos (indiscutiblemente es el : caso del propio Derek Zoolander), pero este tipo de arquetipo es típico en las comedias americanas y, como ya se ha menciona: do, el enfoque de ambas propuestas es muy diferente como para considerar una influencia.

En "Tropic Thunder" (Stiller, 2008) apreciamos esta autoparodia o - crítica al típico cine bélico americano. Los personajes tienen va: lores morales y saben distinguir lo que está bien y lo que está mal : hasta en las situaciones en las que se evidencia el humor. Además, : hay un mayor uso de estereotipos, como puede apreciarse en los : actores que interpretan a las estrellas de Hollywood en esta pelí- cula. Es el caso del actor interpretado por Jack Black, drogadicto : y dedicado a hacer películas de comedia grosera. En "Zoolander" : (Stiller, 2001) pasa algo similar: los modelos son caracterizados de - acuerdo a los estereotipos y por eso son torpes y superficiales.

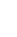

- Stiller tiene un estilo que le permite trocar la perspectiva que se : tiene sobre el personaje tonto ya que, hasta cierto punto, es posible : empatizar con él. El ejemplo más claro está en la construcción de 
Derek Zoolander durante el film. El protagonista reflexiona sobre su vida y se pregunta si le satisface lo que hace, tiene problemas en sus relaciones familiares y quiere ayudar a las personas. Todo : eso forma parte de la personalidad de Derek y permite construir : un personaje con sentido pues sus acciones dentro de la película están justificadas.

Los hermanos Zucker y Jim Abrahams utilizan el elemento del humor absurdo para idiotizar a sus personajes desde el punto de : vista moral y utilizan escenas sin sentido, por lo que existe un contraste entre la realidad verosímil y lo desconocido con relación a lo incoherente. En "Airplane!" (Abrahams, Zucker y Zucker, 1980) : absolutamente nadie reacciona de manera adecuada frente a las situaciones y el slapstick se desarrolla en situaciones de violencia: con golpes rápidos para crear un efecto de gracia en el público que sobrepasa los bordes de la sensatez.

Lo que sí es posible identificar como un elemento común en todas las películas mencionadas en que se tratan de parodias: "Air- : plane!" (Abrahams, Zucker y Zucker, 1980) lo es de las películas de desastre, "The Naked Gun: From the Files of Police Squad!" (Zucker, 1988) del cine policial, "Zoolander" (Stiller, 2001) del mundo del modelaje y del género de espionaje y conspiración y "Tropic Thunder" (Stiller, 2008) lo es de la propia industria cinematográfica y de las películas de guerra.

Por otro lado, mientras que en las películas de los hermanos $\mathrm{Zu}-$ cker los chistes funcionan de manera independiente a la narrativa de la película, el humor en las películas de Stiller carece de sentido si no se conocen antes a los personajes. Por ello sus tramas resultan más complejas: "Zoolander" (Stiller, 2001) es la historia de un : supermodelo idiota en decadencia que se convierte en una máquina asesina que debe acabar con la vida del primer ministro de Malasia y "Tropic Thunder" (Stiller, 2008) nos habla de un grupo de actores que van a Vietnam para grabar una película pero por error son considerados soldados americanos en busca de ataque.

\section{CONCLUSIÓN}

Los chistes en el cine de los hermanos Zucker no dependen de la : narrativa del film porque no se elaboran en base a la historia y se pueden aplicar fuera de la película porque se entienden. En el caso de las películas de Ben Stiller, los momentos cómicos se construyen en función del contexto, la personalidad de los personajes y : las situaciones, lo que muestra una clara diferencia entre ambas propuestas.

\section{SGRUPULOS}

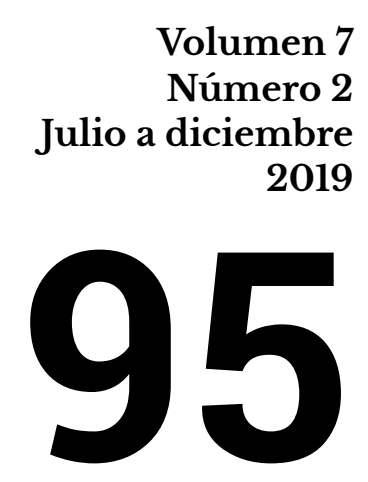

Los hermanos Zucker

y Jim Abrahams utilizan el elemento del humor absurdo para idiotizar a sus personajes desde el punto de vista moral y utilizan escenas sin sentido, por lo que existe un contraste entre la realidad verosímil y lo desconocido con relación a lo incoherente. 
scrupuLOS

\section{Volumen 7}

Número 2

Julio a diciembre 2019

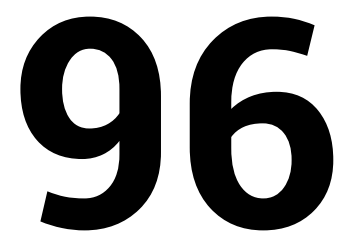

\section{: Referencias}

De lo absurdo a lo paródico, la comedia norteamericana ha dejado de tener un correlato en lo real para internarse en la fantasía más desternillante y todavía es - capaz de proporcionarnos momentos agradables que van más allá del facilismo - escatológico.

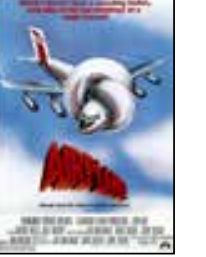

Davison, J. (Productor) y Abrahams, J., Zucker, D., Zucker, J. (Directores). (1980). Airplane! [Película] Estados Unidos: Howard W. Koch Productions.

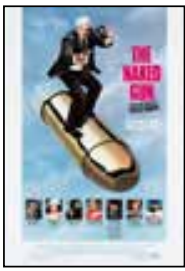

Weiss, R. (Productor) y Zucker, D. (Director). (1988). The Naked Gun: From the Files of Police Squad! [Película]. Estados Unidos: Paramount Pictures.

Cornfeld, S., Rudin, S. (Productores) y Stiller, B. (Productor y Director). (2001). Zoolander [Película]. Alemania y Estados Unidos: Scott Rudin Productions, Red Hour Films, MFP Munich Film Partners GmbH \& Company I. Produktions

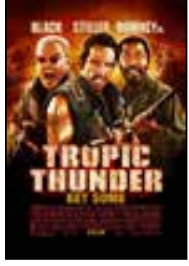

Cornfeld, S., McLeod, E. (Productores) y Stiller, B. (Productor y Director). (2008). Tropic Thunder [Película]. Estados Unidos, Inglaterra y Alemania: Red Hour Films, Goldcrest Pictures e Internationale Filmproduktion Stelladel-Sud Second. tions y Tenth Planet Productions.

Heredero del ZAZ: el cine de Jim Abrahams y de los hermanos Zucker en las comedias de Ben Stiller

Referencia del artículo en APA: Chávez, S., Gómez, S., Montoya, D., Sánchez, A., Torres, C. (2019). Heredero del ZAZ: el cine de Jim Abrahams y de los hermanos Zucker en las comedias de Ben - Stiller. CineScrúpulos, 7(2), 91-96.
Heir of ZAZ: Jim Abrahams and Zucker brothers's cinema in Ben Stiller's comedies

Article reference in APA: Chávez, S., Gómez, S., Montoya, D., Sánchez, A., Torres, C. (2019). Heir of ZAZ: influence of Jim Abrahams and Zucker brothers's cinema in Ben Stiller's comedies. CineScrúpulos, 7(2), 91-96.

CineScrúpulos / Revista digital de diálogo cinematográfico/ ISSN: 2709-0493

- (c) Los autores. Este artículo es publicado por la revista CineScrúpulos del Programa

- Académico de Comunicación Audiovisual y Medios Interactivos de la Facultad de

- Comunicaciones, Universidad Peruana de Ciencias Aplicadas. Este es un artículo de

- acceso abierto, distribuido bajo los términos de la LicenciaCreativeCommons Atribución-

- Compartirlgual 4.0 Internacional (http://creativecommons.org/licenses/by-sa/4.0/), que

- permite el uso no comercial, distribución y reproducción en cualquier medio, siempre que

- la obra original sea debidamente citada. 